\title{
Overmodulation algorithm for space vector modulated inverters and its application to low switching frequency PWM techniques
}

\author{
G.Narayanan and V.T.Ranganathan
}

\begin{abstract}
An overmodulation algorithm is applied to four recently proposed space vector-based synchronised PWM strategies, to extend their range of operation and enhance the DC bus utilisation. The waveform symmetries and the proportionality between the reference magnitude and the fundamental voltage generated are also maintained in the overmodulation zone. It is shown that the relationship between the control variable and the fundamental voltage generated differs widely with the strategy and the pulse number used in low switching frequency PWM techniques unlike that at high switching frequencies. As a result the boundaries between any two consecutive zones of modulation and the maximum modulation index also vary with the strategy and the pulse number used. Cases where operation is possible up to the six-step mode are distinguished from those cases where it is not. The performance of the different strategies is evaluated. It is shown theoretically as well as experimentally that the bus-clamping PWM strategies perform better than the conventional space vector strategy in the overmodulation zone.
\end{abstract}

\section{Introduction}

Voltage source inverter (VSI) fed induction motors are widely used as variable speed drives. PWM patterns are required to be generated for switching the devices in a VSI. There are two popular approaches to real-time generation of PWM patterns, namely the triangle-comparison (TC) approach $[1-6]$ and the space vector (SV) approach [I-91. It was held that the two approaches were equivalent $[4,5]$, but of late, it has been pointed out that the SV approach offers additional flexibilities to the design of PWM strategies over the classical TC approach [8]. Using these flexibilities, a few space vector-based synchronised PWM strategies for high power drives have been proposed recently [8]. These strategies result in reduced harmonic distortion over comparable strategies at low switching frequencies $[8,9]$.

Apart from reduction in the harmonic distortion, PWM techniques for high power drives must also ensure a high DC bus utilisation [10]. This paper investigates the extension of operation of the recently proposed space vectorbased synchronised PWM strategies so as to increase the maximum fundamental voltage generated with a given DC bus voltage. The operation is extended up to the six-step mode where possible. The waveform symmetries are preserved in the overmodulation zone as well. The modulator gain is kept constant in the whole range of modulation.

\section{Triangle-comparisonapproach and space vector approachto real-time PWM}

In the TC approach, three-phase modulating waves are compared against a common triangular carrier to generate the switching instants of the three legs in an inverter. Sinusoidal waves are the most common modulating waves. Suitable measures of triplen frequency components can be added to the three-phase sinusoids to generate different modulating waves with different spectral properties [1-51. Overmodulation with such PWM techniques has been discussed $[11,12]$. The proportionality between the reference magnitude and the fundamental voltage generated can be maintained in the overmodulation zone also by suitably reshaping the modulating waves [12].

A voltage source inverter and the voltage vectors produced by it are shown in Figs. $l a$ and $b$, respectively. In the $\mathrm{SV}$ approach, the voltage reference is provided as a revolving space vector as shown in Fig. $l b$. The revolving reference vector is sampled once in every subcycle $T_{s}$. Given a sample of the reference vector in sector I, with magnitude $V_{R E F}$ and angle $\alpha$ as shown in Fig. $1 b$, the durations for which the active state 1 , the active state 2 , and the two zero states together must be applied in the given subcycle to maintain the volt-second balance are given by $T_{1}, T_{2}$ and $T_{z}$, respectively, in eqn. 1 .

$$
\begin{aligned}
T_{1} & =T_{S} * V_{R E F} * \sin \left(60^{\circ}-\alpha\right) / \sin 60^{\prime \prime} \\
T_{2} & =T_{S} * V_{R E F} * \sin (\alpha) / \sin 60^{\circ} \\
T_{Z} & =T_{S}-T_{1}-T_{2}
\end{aligned}
$$

Sequence $\mathbf{0 1 2 7}$ or $\mathbf{7 2 1 0}$ is commonly used to generate a given average vector in sector I with $T_{z}$ divided between the zero states $\mathbf{0}$ and 7. Use of only one zero state results in the clamping of one of the phases to one of the DC buses. When both zero states are used an equal division of $T_{z}$ leads to lesser ripple current than an unequal division [3]. 
This apportioning of $T_{z}$ between the two zero states in the $\mathrm{SV}$ approach is equivalent to the addition of triplen frequency components in the classical TC approach $[4,5]$. This equivalence has been exploited in the literature to make the PWM generation simpler $[6,7]$.
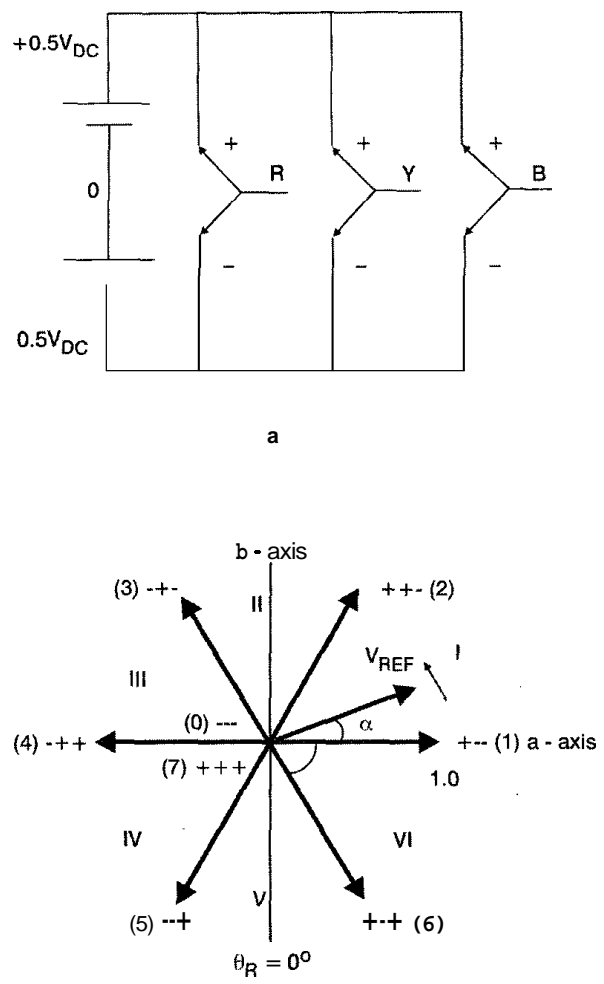

Fig.1 Voltagesource inverter and voltage vectorsproduced by it $a$ Voltage source inverter

$b$ Voltage vectors produced by inverte

$\theta_{R}=$ angle of R-phase fundamental voltage

Sequence 0121, 1210, 7212 or 2127, with either $T_{1}$ or $T_{2}$ divided into two equal halves, can also be used to generate the commanded average vector. With such a sequence: a phase switches more than once in a subcycle, which is not possible in a half carrier cycle in the TC approach. Thus, this is an additional degree of freedom available in the SV approach, and does not have any equivalence in the TC approach [8]. Such additional flexibilities in the SV approach have been exploited in the design of space vectorbased synchronised PWM techniques, proposed recently, to reduce the harmonic distortion in high power drives $[8,9]$. In the present work, modulation index $M$ is defined as the ratio of fundamental voltage generated to the fundamental voltage corresponding to six-step operation with the same DC bus voltage. In the SV approach, $M$ and $V_{R E F}$ are proportional in the linear modulation zone at fairly high switching frequencies as given by eqn. 2 .

$$
M=(0.907 / 0.866) * V_{R E F}
$$

A few overmodulation algorithms have been proposed for space vector modulated inverters in the literature [13-15]. A new overmodulation algorithm is proposed and is applied to space vector-based synchronisecl PWM strategies.

\section{Space vector-based synchronised PWM strategies}

At low switching frequencies the PWM waveform generated must be synchronised with its own fundamental to avoid subharmonics. The waveform must possess half-wave symmetry (HWS) to avoid even harmonics, and threephase symmetry (3PS) to ensure every frequency component is balanced. Further, it is desirable to maintain quarter-wave symmetry (QWS) as well.

To maintain the waveform symmetries there must be an equal number of samples and at identical locations in every sector (i.e. the sampling frequency must be an integral multiple of six times the fundamental frequency), and similar sequences must be used to generate the samples at identical locations in different sectors (e.g. 0127 in sector I, 7230 in sector II, $\mathbf{0 3 4 7}$ in sector III and so on.). Four synchronised PWM strategies based on such a framework are briefly explained as follows [8].

3.1 Conventional space vector strategy (CSVS) In the conventional space vector strategy (CSVS), the number of samples per sector $N$ must be odd to maintain the waveform symmetries. The positions of samples and the sequences used to generate them in sector I are tabulated in Table 1 for $N=3,5,7$. The ratio of the switching frequency to the fundamental frequency, or the pulse number, $P=3 N$ in CSVS [8].

\subsection{Basic bus clamping strategy (BBCS)}

In the basic bus clamping strategy (BBCS), $N$ must be odd as in CSVS. The positions of the samples and the sequences used to generate them in sector I are given in Table 2 for

Table 1: Conventional space vector strategy

\begin{tabular}{llll}
\hline $\begin{array}{l}\text { Samples per } \\
\text { sector } N\end{array}$ & Positions of samples $a$ (deg) & Sequences used in sector I & $\begin{array}{l}\text { Pulse } \\
\text { number } P\end{array}$ \\
\hline 3 & $10,30,50$ & $7210,0127,7210$ & 9 \\
5 & $6,18,30,42,54$ & $0127,7210,0127,7210,0127$ & 15 \\
7 & $4.3,12.9,21.4,30,38.6,47.1,55.7$ & $7210,0127,7210,0127,7210,0127,7210$ & 21 \\
\hline
\end{tabular}

Table 2: Basic bus clamping strategy

\begin{tabular}{lllll}
\hline $\begin{array}{l}\text { Samples per } \\
\text { sector } N\end{array}$ & Positions of samples $a$ (deg) & Sequences used in sector I & $\begin{array}{l}\text { Pulse } \\
\text { number } \boldsymbol{P}\end{array}$ & $\begin{array}{l}\text { Type of } \\
\text { clamping }\end{array}$ \\
\hline 3 & $10,30,50$ & $127,7210,012$ & 7 & $60 "$ \\
5 & $6,18,30,42,54$ & $721,127,7210,012,210$ & 11 & $60 "$ \\
5 & $6,18,30,42,54$ & $012,210,0127,721,127$ & 11 & $30 "$ \\
7 & $4.3,12.9,21.4,30,38.6,47.1,55.7$ & $127,721,127,7210,012,210,012$ & 15 & $60 "$ \\
\hline
\end{tabular}


$N=3,5,7$. For $N=5,9,13 \ldots$ two sets of sequences are possible to generate the samples (as shown for $N=5$ in Table 2). One set of sequences leads to clamping of every phase during the middle 60" duration of every half cycle of its fundamental voltage as shown in Fig. $2 a$. This is called '60" clamping'. The other set of sequences results in the clamping of every phase during the middle 30 " duration of every quarter cycle of its fundamental as shown in Fig. 26. This is called ' $30^{\circ}$ clamping'. On the other hand, for $N=3$, $7,11 \ldots$ only one set of sequences leading to 60 " clamping is possible (as shown for $N=3$ and 7 in Table 2). In this strategy, $P=(2 N+1)[9]$.
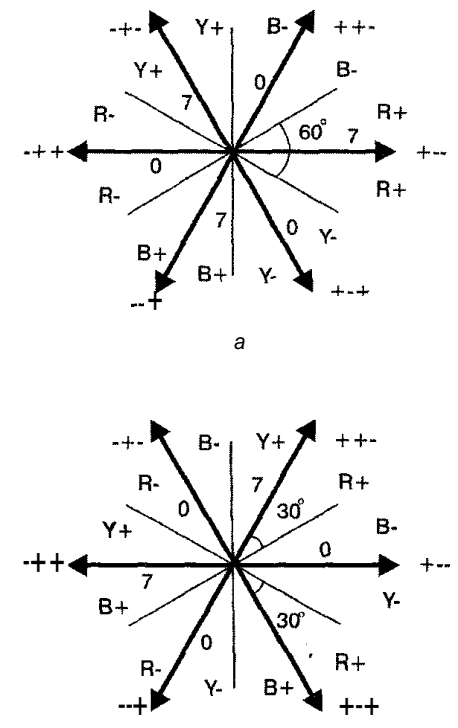

$\boldsymbol{b}$

Fig.2 Types of clamping $a 60^{\circ}$ clamping
$b 30^{\circ}$ clamping

\subsection{Boundary sampling strategy (BSS)}

A sample along the boundary between sector VI and sector I can be generated using the sequence $\mathbf{0 1 0}$ or 101, dividing either $T_{0}$ or $T_{1}$ into two equal halves. Such boundary samples are used in the boundary sampling strategy (BSS) as shown in Table 3. Here, $N$ is even, and $P=(2 N+1)$ [8].
3.4 Asymmetric zero-changing strategy(AZCS)

The zero state used is changed over the middle two subcycles in the asymmetric zero-changing strategy. Sequences 0121, 127 or 7212, 210 are used for the middle two samples. $N$ is even in this strategy, and $P=2 N+1$. With $N=$ $2,6,10 \ldots$ two types of sequences are possible (as shown for $N=2$ and 6 in Table 4), one leading to $60^{\circ}$ clamping and the other to $30^{\prime \prime}$ clamping. With $N=4,8,12 \ldots$ only $60^{\prime \prime}$ clamping is possible (as shown for $N=4$ in Table 4) [8].

\section{Proposed overmodulationalgorithm}

If modulation is carried out as given in Section 3, the average vectors generated over different subcycles in a cycle at steady state are of equal lengths and are uniformly spaced as shown in Fig. $3 a$. The tip of the average vector generated has a circular trajectory of radius $V_{C I R}$. Hence this zone is termed here as the 'circular modulation zone'. The modulation index $M$ is increased by increasing $V_{C I R}$. The upper bound of this zone is when the tips of the average vectors in the middle of every sector touch the hexagon. To increase $M$ further, the average vectors generated over the different subcycles must be of unequal lengths or nonuniformly spaced, or both. This zone of modulation is called the 'overmodulation zone'.

Bolognani et al. consider the whole overmodulation zone to be a single undivided zone [13]. The more popular approach is to divide the overmodulation zone into two zones, namely 'overmodulation zone-I' and 'overmodulation zone-II' $[14,15]$. The two-zone approach to overmodulation results in lesser harmonic distortion than the onezone approach [15], and hence, is taken up in the present work. In overmodulation zone-I, the trajectory of the tip of the average vector generated is partly circular and partly hexagonal as shown in Fig. 36. In overmodulation zone-11, the trajectory is fully hexagonal as shown in Fig. 3c. The average vectors over the different subcycles are of unequal lengths, but are uniformly spaced in zone-I. They are of unequal lengths and are also nonuniformly spaced in zone11. With the one-zone overmodulation algorithm, they are of equal lengths but are nonuniformly spaced [13].

During overmodulation, in every subcycle the sample of the reference vector must first be corrected before being used for PWM calculations as shown in Fig. 4a. Such correction or transformation is termed as 'premoddation' [13-15]. Given $V_{R E F}$ and $a$, the magnitude $\left(V_{P}\right)$ and the

Table 3: Boundary sampling strategy

\begin{tabular}{lllll}
\hline $\begin{array}{l}\text { Samples per } \\
\text { sector } N\end{array}$ & Positions of samples $\alpha$ (deg) & Sequences used in sector $\mathbf{r}$ & $\begin{array}{l}\text { Pulse } \\
\text { number } \boldsymbol{P}\end{array}$ & $\begin{array}{l}\text { Typeof } \\
\text { clamping }\end{array}$ \\
\hline 2 & 0,30 & 010,0127 & 5 & $30^{\circ}$ \\
4 & $0,15,30,45$ & $101,127,7210,012$ & 9 & $60^{\prime \prime}$ \\
6 & $0,10,20,30,40,50$ & $010,012,210,0127,721,127$ & 13 & $30^{\prime \prime}$ \\
\hline * with a small discontinuity in the middle of the clamping duration
\end{tabular}

Table 4. Asymmetric zero-changing strategy

\begin{tabular}{lllll}
\hline $\begin{array}{l}\text { Samples per } \\
\text { sector } \boldsymbol{N}\end{array}$ & Positions of samples $a$ (deg) & Sequences used in sector 1 & $\begin{array}{l}\text { Pulse } \\
\text { number } \boldsymbol{P}\end{array}$ & $\begin{array}{l}\text { Typeof } \\
\text { clamping }\end{array}$ \\
\hline 2 & 15,45 & 7212,210 & 5 & $60^{\prime \prime}$ \\
2 & 15,45 & 0121,127 & 5 & $30^{\circ}$ \\
4 & $7.5,22.5,37.5,52.5$ & $127,7212,210,012$ & 9 & $60^{\prime \prime}$ \\
6 & $5,15,25,35,45,55$ & $721,127,7212,210,012,210$ & 13 & $60^{\prime \prime}$ \\
6 & $5,15,25,35,45,55$ & $012,210,0121,127,721,127$ & 13 & $30^{\circ}$ \\
\hline
\end{tabular}




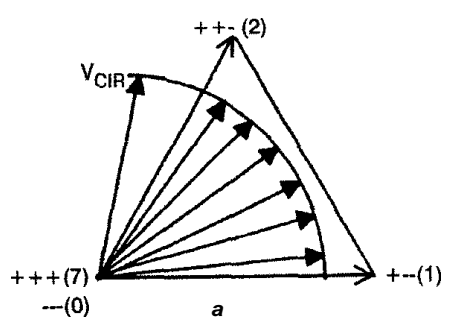

Fig.3 Different zones of modulation

a Circular modulation zone

$b$ Overmodulationzone-I

$c$ Overmodulationzone-II

angle $\alpha_{P}$ of the premodulated sample are calculated. $V_{P}$ and $\alpha_{P}$ are then used (instead of $V_{R E F}$ and $a$ ) in eqn. 1 to calculate the inverter state times. The premodulation procedures adopted in the two zones in the proposed overmodulation algorithm are described here.

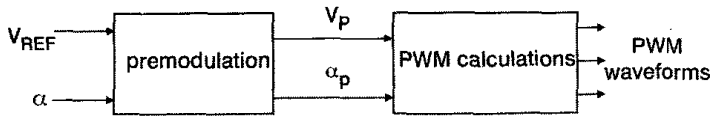

a

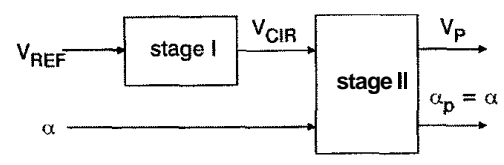

b

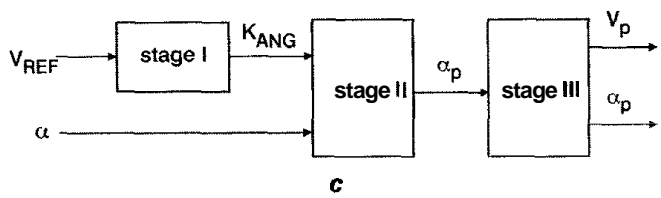

Fig.4 Premodulation for overmodulation and linearity

a Premodulation before PWM calculations

$b$ Premodulation in overmodulationzone-I

c Premodulation in overmodulation zone-I1

In overmodulation zone-I, $\boldsymbol{A}$ is increased by increasing $V_{C I R}$. Stage I of premodulation involves obtaining the appropriate value of $V_{C I R}$ for a given $V_{R E F}$ as shown in Fig. 4b. Given $V_{C I R}$ and $\alpha$, the magnitude $V_{P}$ and the angle $\alpha_{P}$ of the premodulated sample are calculated using eqn. 3 in the stage-I1 as illustrated in Fig. 4b. Zone I ends when the tips of all the average vectors generated touch the hexagon.

$$
\begin{aligned}
V_{P(M A X)} & =0.866 / \cos \left(30^{\circ}-\alpha\right) \\
V_{P} & =V_{C I R}, \quad \text { if } V_{C I R} \leq V_{P(M A X)} \\
& =V_{P(M A X)}, \quad \text { if } V_{C I R}>V_{P(M A X)} \\
\alpha_{P} & =a !
\end{aligned}
$$

In overmodulation zone-11, $M$ is increased by shifting the average vectors generated closer to their nearest sector boundaries. In the existing two-zone algorithm, premodulation is carried out differently for samples around sector boundaries and for those around the centres of sectors $[14,15]$. It will be simpler and more convenient to adopt a uniform policy towards all the samples in a sector, especially when the number of samples is low as in high power drives. In the present work, the angle between the given sample and its nearest sector boundary is multiplied by a fraction called the 'angle correction factor' $K_{A N G}$. The new angle gives the position of the premodulated sample. The maximum length of the average vector possible at this angle is taken as the magnitude of the premodulated sample. $K_{A N G}$ is decreased from 1 towards 0 to increase $M$ in zone-11.

The premodulation procedure in zone-I1 consists of three stages as shown in Fig. $4 c$. During stage-I the appropriate value of $K_{A N G}$ is obtained for a given $V_{R E F}$. During stageII, $\alpha_{P}$ is calculated using $K_{A N G}$ and $\alpha$ as per eqn. 4. In stage-111, $V_{P}$ is calculated using $\alpha_{P}$ as given in eqn. 4 .

$$
\begin{array}{rlrl}
\alpha_{P} & =K_{A N G}{ }^{*} \alpha, & & 0^{\prime \prime} \leq a^{\prime}<30^{\circ} \\
& =30^{\circ}, & \alpha=30^{\circ} \\
& =60^{\prime \prime}-K_{A N G} *\left(60^{\circ}-\alpha\right), \mathbf{3 0}^{\prime \prime}<\alpha<60^{\prime \prime} \\
V_{P} & =0.866 / \cos \left(30^{\circ}-\alpha_{P}\right) &
\end{array}
$$

The relationships $V_{C I R}$ against $V_{R E F}$ and $K_{A N G}$ against $V_{R E F}$ are required during stage-I of premodulation in zoneI and zone-11, respectively. $V_{C I R}$ against $V_{R E F}$ can be obtained using $M$ against $V_{C I R}$ and eqn. 2. Similarly, $K_{A N G}$ against $V_{R E F}$ can be derived from $M$ against $K_{A N G}$ and eqn. 2. Hence the variation in $M$ against $V_{C I R}$ in zone-I and against $K_{A N G}$ in zone-I1 must first be studied. This is carried out in the following Section for the four synchronised PWM strategies and for different values of $N$.

\section{Operation of synchronised PWM strategies in overmodulation zone}

Overmodulation zone-I begins when the tip of the middle average vector in every sector just touches the hexagon in case of CSVS, BBCS and BSS, i.e. at $V_{C I R}=0.866$. With further increase in $V_{C I R}$, the length of this average vector does not increase; in other words, this average vector saturates. However, the lengths of the other average vectors continue to grow. The next pair of average vectors, one on either side of the middle average vector, saturates at $V_{C I R}=$ $0.866 / \cos \left(\omega T_{s}\right)$, where $\omega=2 \pi F_{1}$ and $F_{1}$ is the fundamental frequency. Pulse dropping begins here. Other pairs of average vectors, if any, saturate at $V_{C I R}=0.866 / \cos \left(2 \omega T_{s}\right)$, $0.866 / \cos (3 \omega T s), \ldots$. This zone ends when the tips of all theaverage vectors touch the hexagon, i.e. at $V_{C I R}=$ $0.866 / \cos \left((\pi / 6)-\omega T_{s} / 2\right)$ in case of CSVS and BBCS, and at $V_{C I R}=1$ in case of BSS which has a sample on every sector boundary.

In the case of AZCS, which has no sample at the centre of the sector, overmodulation zone-I begins when the tips of the middle two average vectors just touch the hexagon, i.e. at $V_{C I R}=0.866 / \cos \left(\omega T_{s} / 2\right)$. With increase in $V_{C I R}$, additional pairs of average vectors saturate at $V_{C I R}=0.866 /$ $\cos \left(3 \omega T_{s} / 2\right), \quad 0.866 / \cos \left(5 \omega T_{s} / 2\right), 0.866 / \cos \left(7 \omega T_{s} / 2\right), \ldots$ until all the average vectors saturate at $0.866 / \cos \left((\pi / 6)-\omega T_{s} / 2\right)$. 
As mentioned earlier, $K_{A N G}$ is decreased from 1 to 0 to increase $M$ in overmodulation zone-TI. In case of AZCS, which has no sample at the centre of sector, every average vector generated gets shifted closer to its nearest sector boundary with decrease in $K_{A N G}$. At the end of zone-11, when $K_{A N G}=0$, all the average vectors generated during the first half of a sector are along the sector boundary on the clockwise side, and those generated during the second half are along the other boundary on the anticlockwise side. The length of every average vector generated is equal to that of the active vectors. Only active vector $\mathbf{1}$ gets applied from the middle of sector VI to that of sector I, active vector $\mathbf{2}$ from the middle of sector I to that of sector II, and so on. Thus, a six-step mode is attained.

In strategies with a sample at the centre of every sector, the middle average vector alone retains it position, while all the other average vectors get shifted closer to their nearest
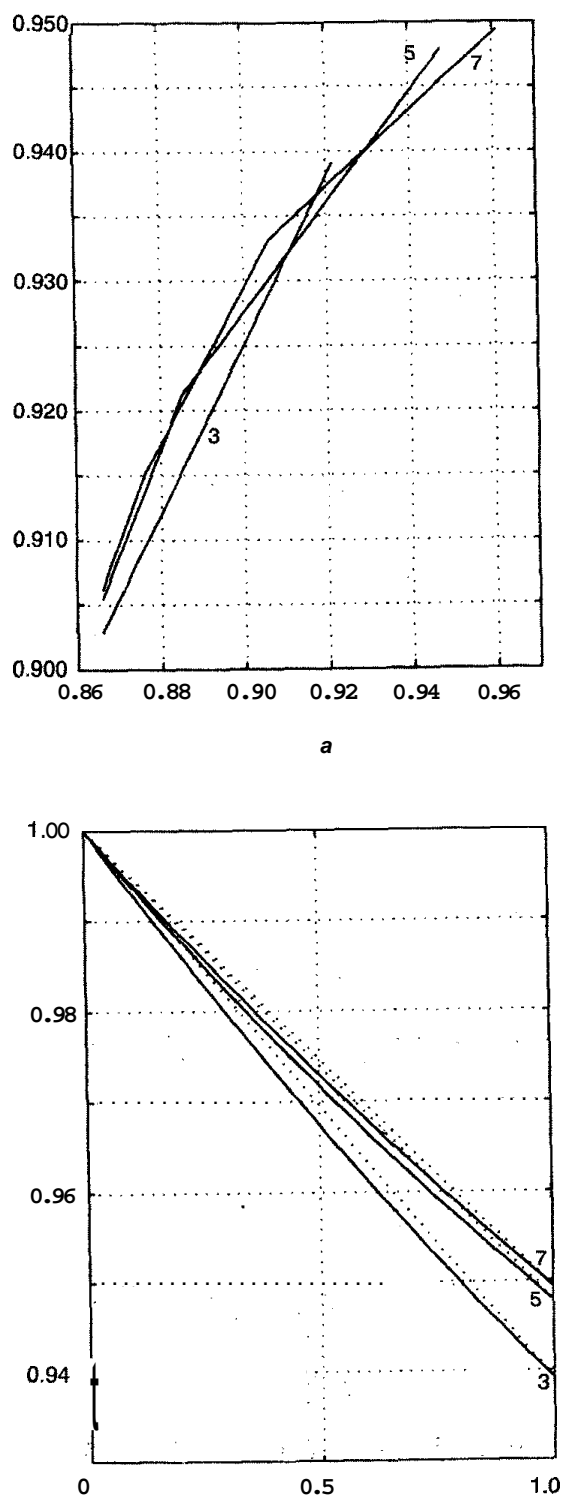

Fig.5 - Voltage control during overmodulation - CSVS a $M$ against $V_{C R}$
$b V_{C R}$ against $V_{R E F}$
$c M$ against $K_{A N G}$
$d K_{A N G}$ against $V_{R E F}$ sector boundaries with decrease in $K_{A N G}$. At $K_{A N G}=0$ when zone-I1 ends, $N-1$ average vectors are generated along every sector boundary and one average vector is generated at the centre of every sector. Thus, active vector $\mathbf{1}$ gets applied in all the subcyles prior to the middle subcycle, and active vector $\mathbf{2}$ in all the subcycles following the middle subcycle in sector I.

In the middle subcycle, $T_{1}=T_{2}=T / 2$. As no zero vector is used in this subcycle, if the sequence corresponding to this sample is 0127 in the circular modulation zone, then the sequence in the overmodulation zone is just 12. This means that the inverter remains in the state 1 during the first half of the subcycle, and hence during the whole first half of the sector. Thus, a six-step mode is attained in the case of 30" clamping.

On the other hand, if the sequence corresponding to the middle sample is $\mathbf{7 2 1 0}$ in the circular modulation zone, the
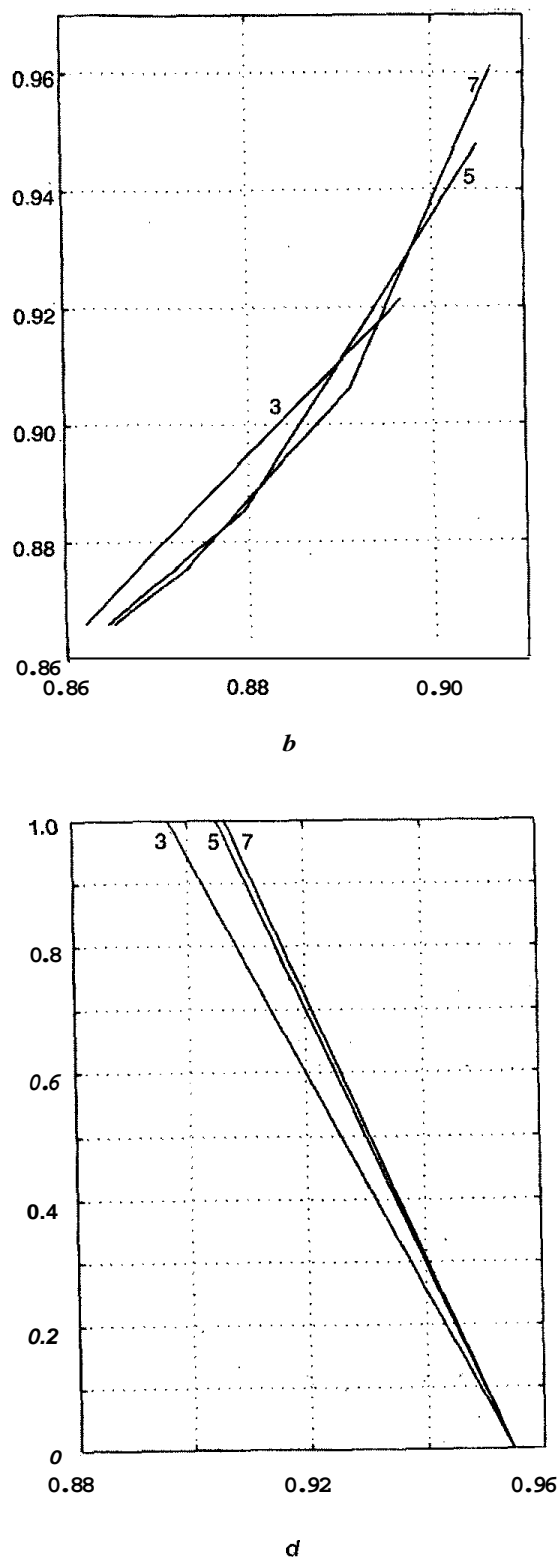
sequence in overmodulation zone is just 21. That is, the inverter is in state $\mathbf{2}$ during the first half of the subcycle and in state $\mathbf{1}$ in the second half of the subcycle. In the earlier subcycles, the inverter is in state $\mathbf{1}$, and the later subcycles it is in state $\mathbf{2}$. There is a switching from $\mathbf{1}$ to $\mathbf{2}$ both at the beginning and the end of the middle subcycle, and one switching from $\mathbf{2}$ to $\mathbf{1}$ at the central instant. Thus, there are three switchings of the Y-phase in sector I. This implies that every phase switches at every zero-crossing of its fundamental voltage, and also at angles $\omega T / 2$ before and after every zero-crossing. Thus, the six-step mode is not attained, and the maximum modulation index $M_{M A X}=2 \cos \left(\omega T_{s} / 2\right)$ -1 . This is the case with $60^{\circ}$ clamping.

Thus, AZCS operates up to a six-step mode. In the case of BBCS and BSS $M_{M A X}=1$ with $30^{\prime \prime}$ clamping, and as previously mentioned with $60^{\circ}$ clamping. Illustrative PWM
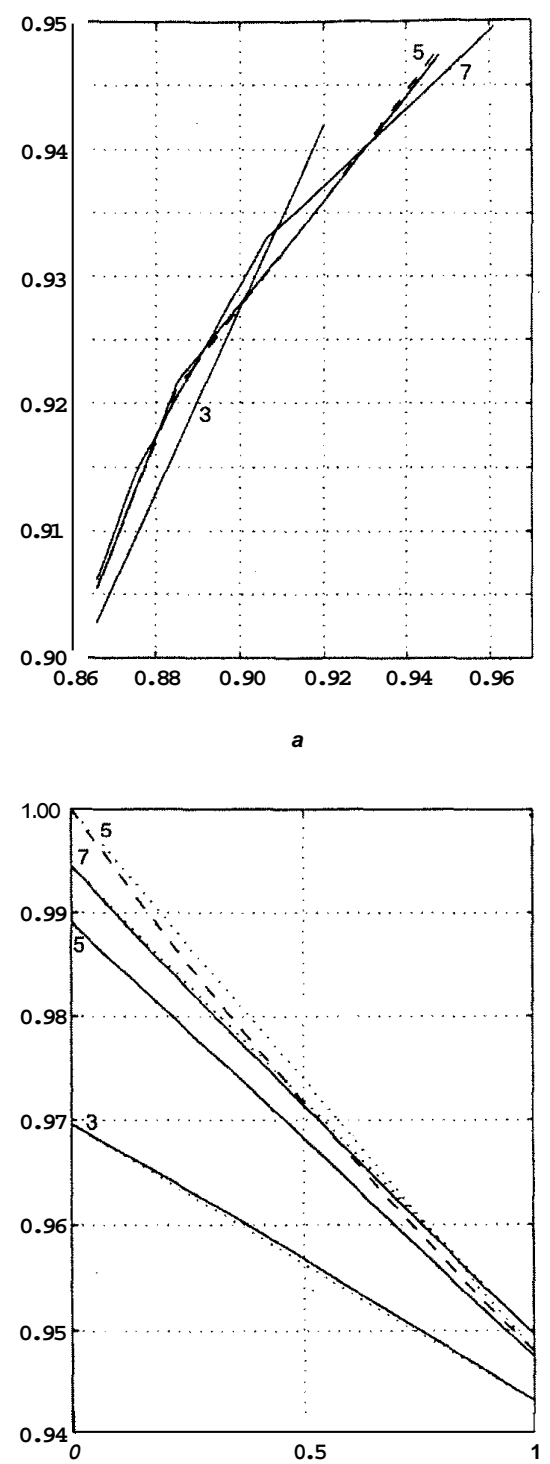

$c$

Fig. 6 Voltage control during overmodulation-BBCS

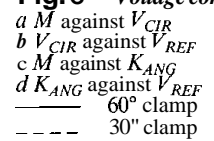

waveforms will be presented in the following Section. In the case of CSVS, with $N=3$ for example, the sequences 7210, 0127 and 7210 are used for the three samples in sector I as shown in Table 2. Thus, the middle sample uses the sequence 0127, and the six-step mode is attained. On the other hand, if the sequences used for the three samples are 0127, 7210, 0127, respectively, the six-step mode cannot be attained here as well.

The voltage control characteristics of CSVS are presented in Fig. 5. The variation in $\boldsymbol{M}$ against $V_{C I R}$ in zone-I is presented in Fig. $5 a$ for CSVS with $N=3,5,7$. The corresponding $V_{C I R}$ against $V_{R E F}$ curves, required for premodulation, are presented in Fig. $5 b$. The variation in $M$ with $K_{A N G}$ in zone-I1 is shown in Fig. $5 c$. For simplicity these curves can be approximated by the dotted straight lines shown. The corresponding $K_{A N G}$ against $V_{R E F}$ curves,
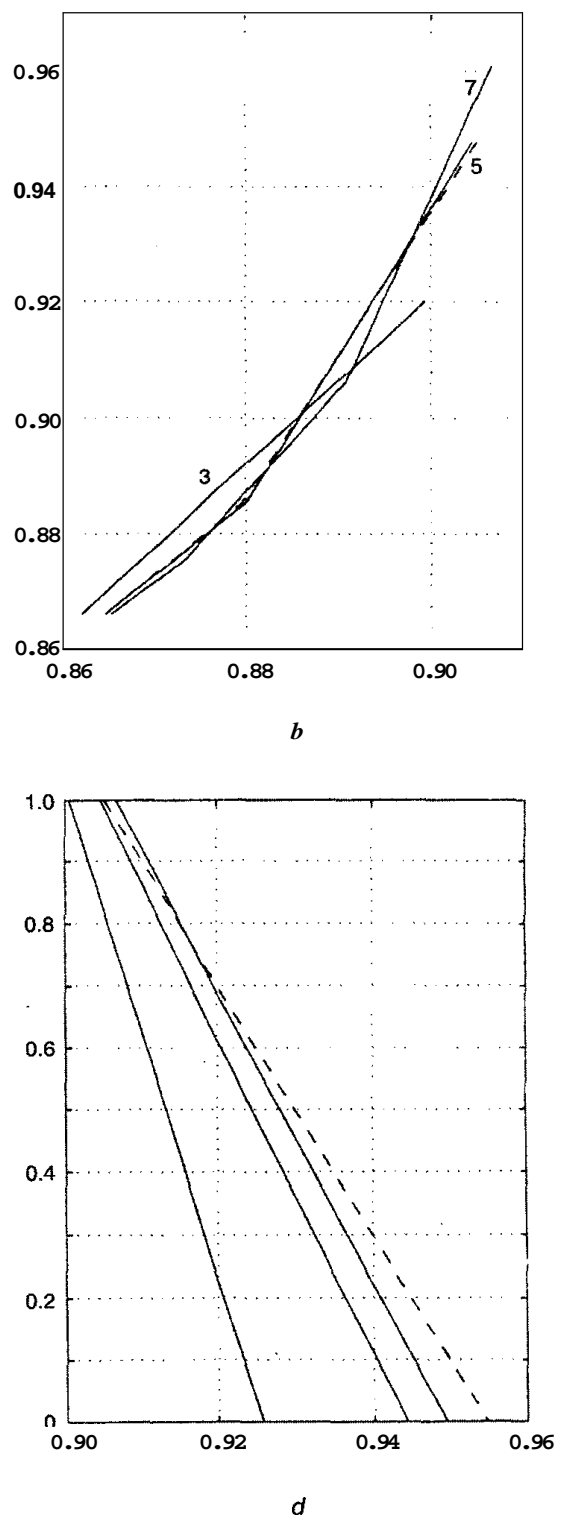
used for premodulation in zone-11, are given in Fig. $5 d$. The voltage control characteristics of BBCS are presented in Fig. 6 for $N=3,5$ and 7. Similar characteristics corresponding to BSS and AZCS are presented in Figs. 7 and 8, respectively, for $N=2,4$ and 6 . In case of BSS with $N=2$, the six-step mode is attained at the end of zone-I itself. In case of $A Z C S$ with $N=2$, the end of circular modulation zone marks the beginning of overmodulation zone- 11 . Hence there is no operation in zone-I.

$M$ against $V_{C I R}$ is piecewise linear for all the strategies. Every time a pair of samples saturates, the rate of rise in $M$ with $V_{C I R}$ becomes lesser. Hence, the slope of the successive linear portions in the $M$ against $V_{C I R}$ is less. These points, at which a pair of samples saturates, can be termed 'breakpoints'. In the present work, no minimum pulse duration is ensured by the PWM algorithms. If any condition on minimum pulse duration is imposed, the break-points shift to slightly smaller values of $M$, and so does pulse dropping.
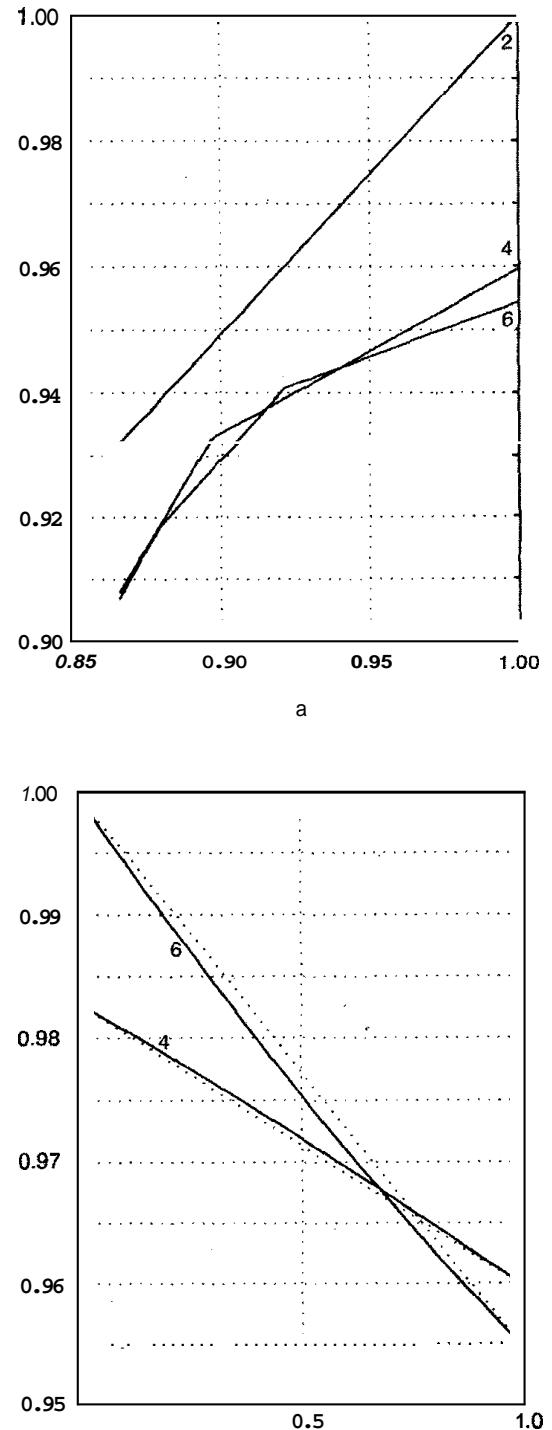

Fig.7 . Voltage control during overmodulation - BSS $a M$ against $V_{C D}$
$b V$ b. $M$ against $K$ age $d K_{A N G}$ against $V_{R E T}$
The $K_{A N G}$ against $M$ characteristics are 'almost' linear for all the strategies. These can all be approximated by the dotted straight lines shown in the respective Figures. For a given value of $K_{A N G}, 30$ " clamping leads to slightly higher $M$ than 60" clamping, wherever both are possible. The control characteristics being piecewise linear or approximately linear helps easy implementation.

Instead of $V_{C I R}, \alpha_{C I R}$ can also be used as the control variable in zone-I (see Fig. 3b) [15]. The relationship between $V_{C I R}$ and $\alpha_{C I R}$ is nonlinear as shown in eqn. 5 .

$$
V_{C I R}=0.866 / \cos \left(30^{\circ}-\alpha_{C I R}\right)
$$

From the $M$ against $V_{C I R}$ characteristics in Figs. 5-8 and from eqn. 5, it can be seen that use of $\alpha_{C I R}$ as the control variable would result in every linear section of the piecewise linear characteristics becoming nonlinear. It can also be shown that with the use of 'hold angle' $\alpha_{H}$ as the control variable and the premodulation procedure as in the existing
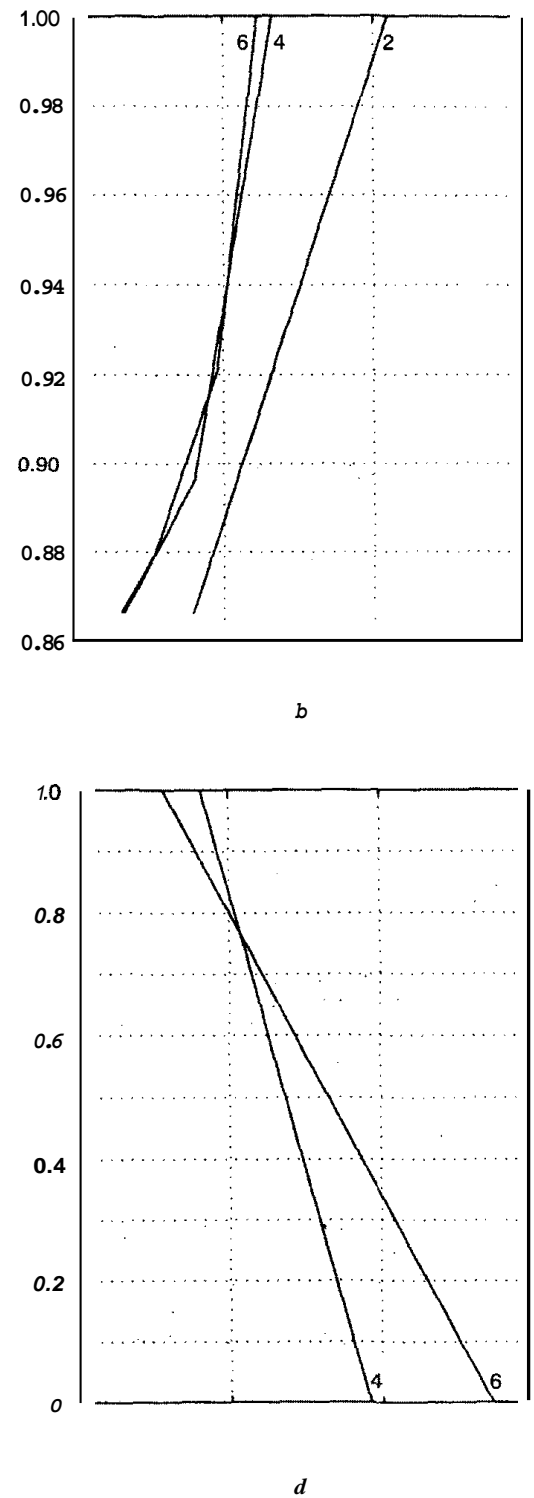

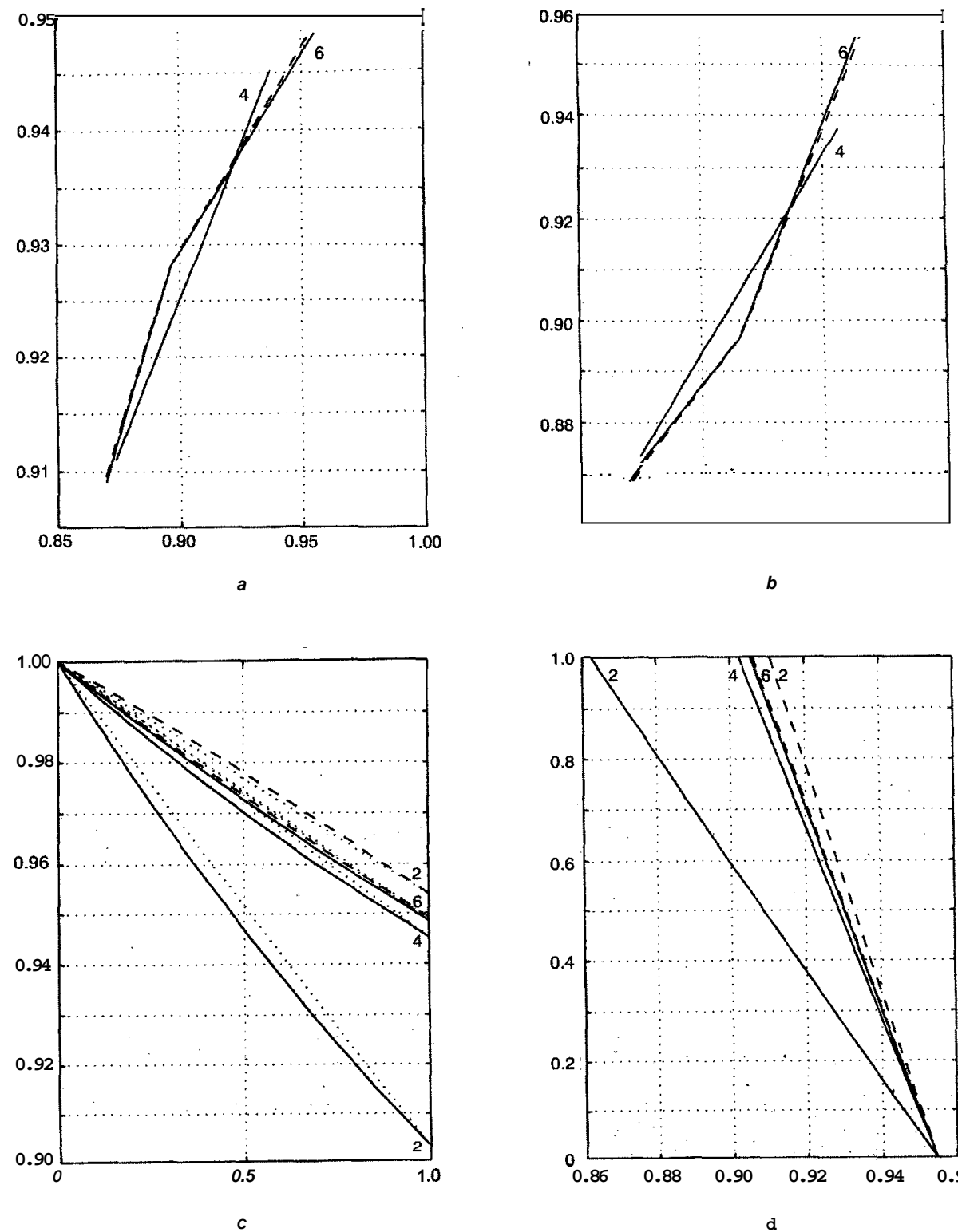

Fig.8 Voltagecontroldurmg overmodulation - AZCS

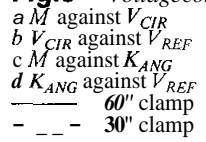

two-zone overmodulation algorithms in zone-I1 [14, 15], the voltage control characteristic in this zone also becomes nonlinear. Thus, the nature of the characteristics would appear to depend on the choice of the control variable and the premodulation procedure adopted (or in other words, the overmodulation algorithm used). But the precise characteristics depend on the number of samples per sector $N$, the position of samples and the sequences used (or in the other words, the strategy used, the value of $N$ and the type of clamping, if any).

Despite the application of the same overmodulation algorithm, the voltage control characteristics are different for different strategies and different values of $N$ as shown in Figs. 5-8. At high switching frequencies, the circular modulation zone ends at $\boldsymbol{A}=0.907$ and overmodulation zone-I ends at $M=0.952[14,15]$. However, at low switch-

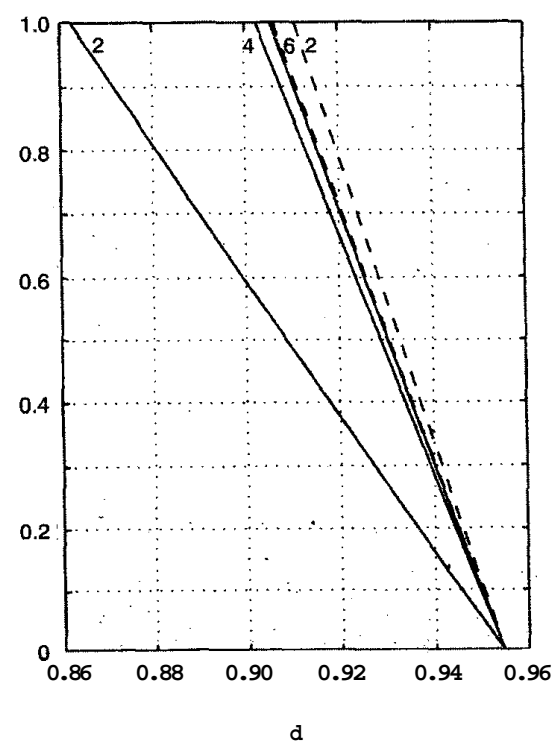

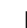

ing frequencies, these boundaries differ with 'the strategy used and the value of $N$.

The relationship between $V_{R E F}$ and $M$ is linear in the circular modulation zone at fairly high switchmg frequencies. However, at very low switchmg frequencies $(\boldsymbol{P}<9)$, this relationship is nonlinear. A suitable premodulation can be used in such cases to ensure the desired proportionality between the two.

\section{Results}

The synchronised PWM strategies are implemented on an INTEL 80C196KB microcontroller. The prototype is a $200 \mathrm{~V}, 3 \mathrm{~kW}, 50 \mathrm{~Hz}$ constant $\mathrm{V} / \mathrm{F}$ induction motor drive fed from a $5 \mathrm{kVA}$ 
are presented in Figs. 9 and 10. One full cycle is shown, starting from the positive zero-crossing of the fundamental voltage. Waveforms pertaining to only one phase are shown, as three-phase symmetry is always maintained. The waveforms shown are at $M=0.92,0.96$ and $M_{M A X}$, demonstrating the operation of the different strategies in the overmodulation zone. The corresponding experimental noload current waveforms are shown in Figs. 11 and 12, respectively. The corresponding measured full-load current waveforms are presented in Figs. 13 and 14, respectively.

Fig. 9 presents the pole voltage waveforms for three cases having a nominal pulse number $\boldsymbol{P}_{N O M}$ of 9 , where nominal pulse number is the pulse number before the onset of pulse dropping. The transition from PWM to six-step mode in the case of CSVS with $\mathrm{N}=3$ is demonstrated by the pole voltage waveforms presented in Figs. $9 a-c$. The waveform symmetries are preserved throughout the overmodulation zone. Similar transition in case of $A Z C S$ with $N=4$ is shown in Figs. $9 d-f$. Pole voltage waveforms corresponding to BSS with $\mathrm{N}=4$ are shown in Figs. $9 g-i$. In the first two cases the pulse number $\boldsymbol{P}$ drops from 9 to 5 at the end of zone-I, and finally to 1 at the end of zone-11. In the third case, $\boldsymbol{P}$ drops from 9 to 5 during the course of zone-I, and then to 3 at the end of zone-I. The pulse number continues to be 3 in the whole of zone-I1 till $M_{\text {MAX }}$. Hence at $M=$ 0.96 , the first two cases have $\boldsymbol{P}=5$, but the third case has $\boldsymbol{P}=3$. Thus, despite having the same nominal pulse number, the pattern of pulse dropping is different for different strategies.

Pole voltage waveforms corresponding to BBCS with $N$ $=5$ and 60" clamping are shown in Figs. $10 a-c$, and those corresponding to BBCS with $N=5$ and 30" clamping are shown in Figs. $10 d f$. Both have a nominal pulse number of 11. In the former case, the pulse number drops from 11
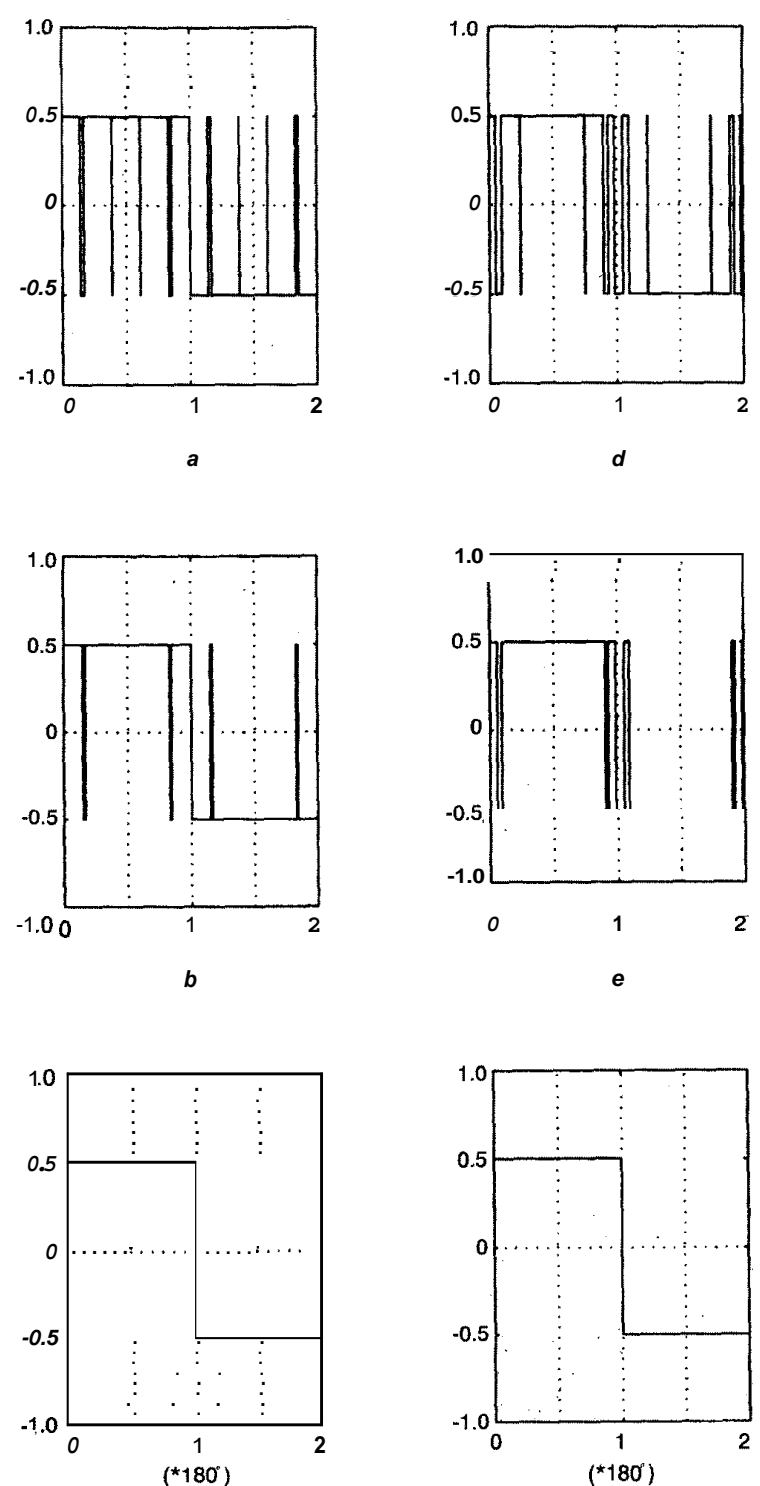

Fig.9 Typical pole voltage waveforms$a-c$ CSVS with $N=3, M=0.92,0.96, M_{\text {MAX }}=1.0$ $d-f$ AZCS with $N=\mathbf{4}, M=0.92,0.96, M_{\text {MAX }}=1.0$ $g-i$ BSS with $N=\mathbf{4}, M=0.92,0.96, M_{\text {MAX }}=0.983$
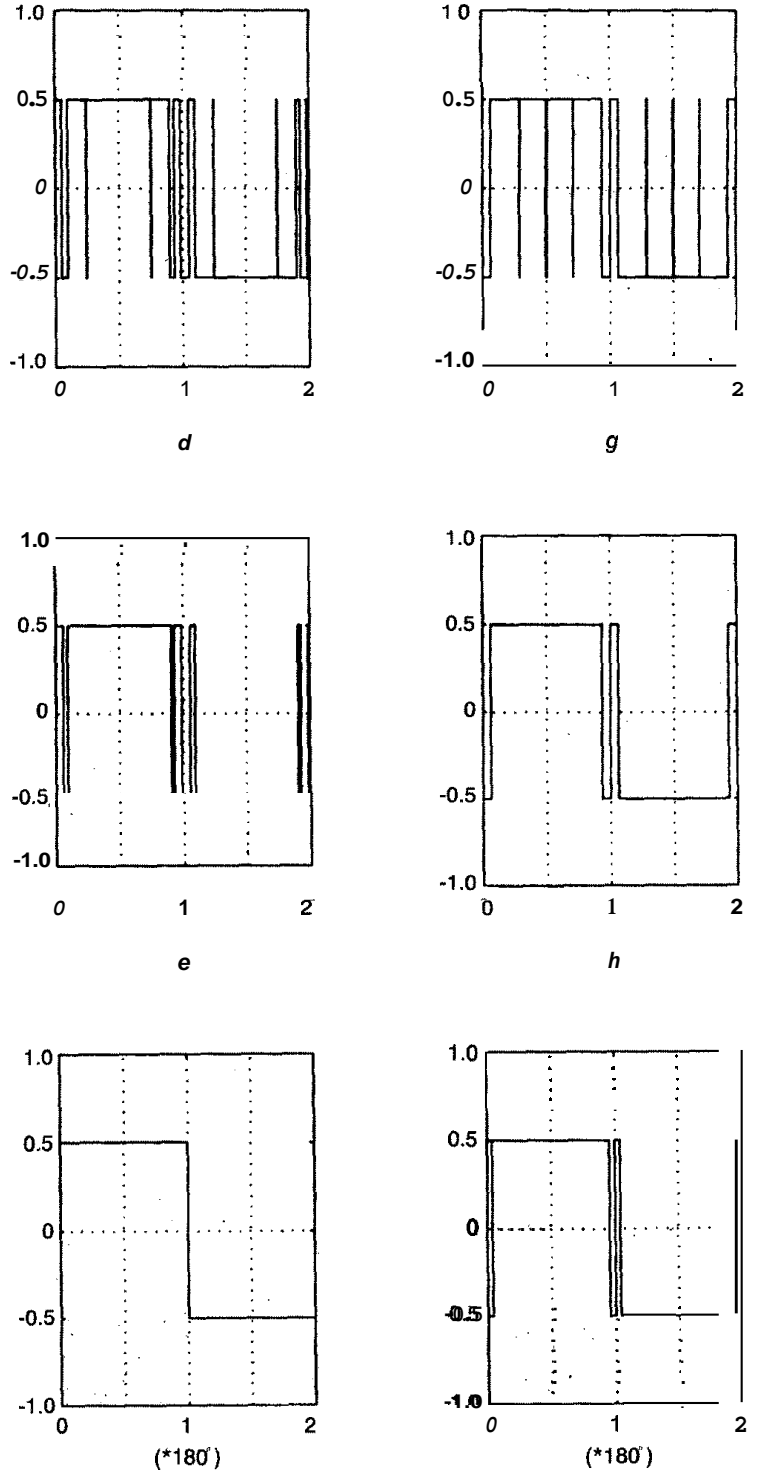

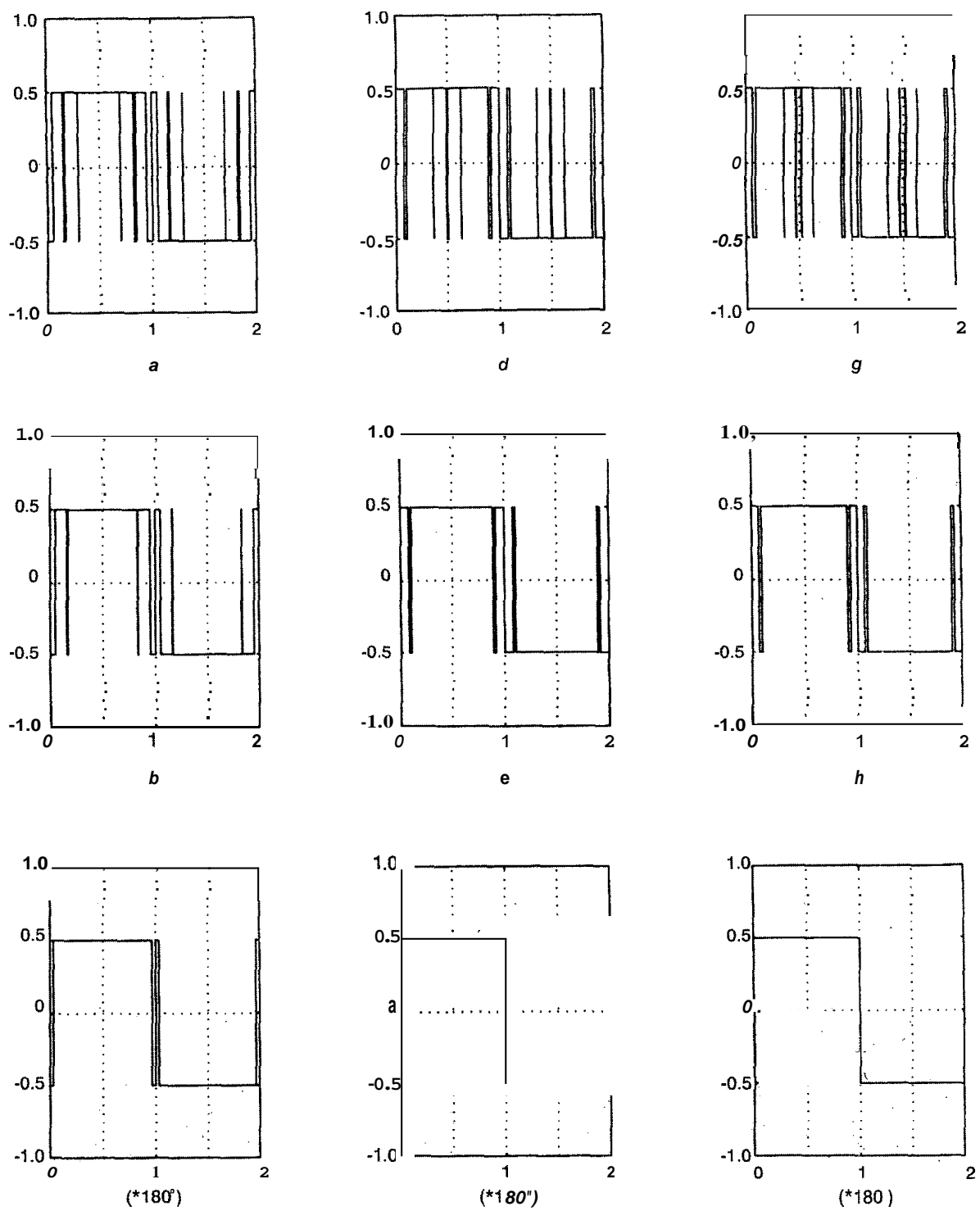

Fig.10 Typical pole voltagewaveforms-II

$$
f
$$

i

to 7 during the course of zone-I, then continues to be 7 till the end of zone-I1 where it finally drops to 3. In the latter case, the pulse number drops from 11 to 7 during the course of zone-I and then to 5 at the end of zone-I. The pulse number finally drops to 1 at the end of zone-11. Thus, the pattern of pulse dropping differs with the type of clamping, even for the same strategy and the same nominal pulse number.

Pole voltage waveforms corresponding to BSS with $N=$ 6 are shown in Figs. $10 g-i$. Here the pulse number drops from 13 to 9 during the course of overmodulation zone-I. It then drops to 5 at the end of zone-I, and finally to 1 at the end of zone-11.

In the case of AZCS, there are no switchings at the zerocrossings of the fundamental voltage, and the PWM waveforms lack QWS around the zero-crossings. In all other cases, the PWM waveforms possess HWS and QWS.
In the case of BSS with $N=4$ and also BBCS with $N=$ 5 and 60 " clamping, every phase switches from the positive bus to the negative bus at the positive zero-crossing of its fundamental voltage and vice versa. In these cases, the sixstep mode is not attained. There are two switchings, one at $\omega T_{s} / 2$ before every zero-crossing and another at $\omega T_{s} / 2$ after every zero-crossing at $M=M_{M A X}$. Hence $M_{M A X}=$ $[2 \cos (\omega T / 2)-1]=\left[2 \cos \left(30^{\circ} / \mathrm{N}\right)-1\right]$. In all other cases presented, every phase switches from the negative bus to the positive bus during the positive zero-crossing of its fundamental voltage and vice versa. Six-step mode is attained eventually. In the former cases where the six-step mode is not attained, the peak ripple current opposes the fundamental current as can be seen in Figs. $11 \mathrm{~g}-\mathrm{i}$ and $12 \mathrm{u}-\mathrm{c}$. These have lower peak currents. In the latter cases where the six-step mode is attained, the peak ripple current adds up to the fundamental current as can be observed in Figs. 1la-c and $12 d-i$. 

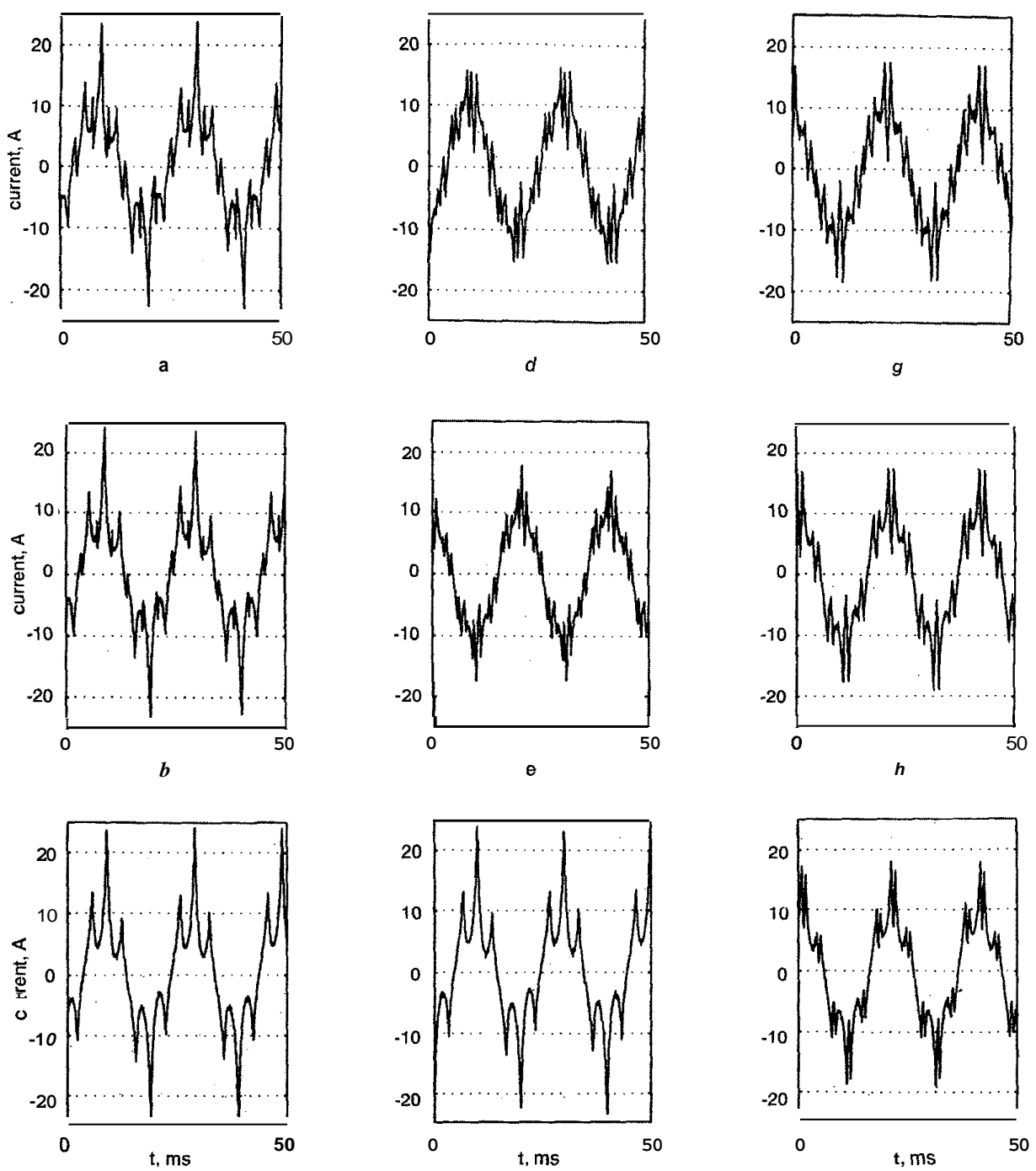

Fig.11 Experimental no-load current waveforms- 1 a c CSVS with $N=3 . M=0.92,0.96, M_{\text {MAX }}=1.0$
$d-f$ AZCS with $N=4, M=0.92,0.96, M_{M A X}=1.0$ d-f $\mathrm{f}$ AZCS with $N=4, M=0.92,0.96, M_{\text {MAX }}=1.0$
$g-i$ BSS with $N=4, M=0.92,0.96, M_{M 4 X}=0.983$

The total harmonic distortion factor $I_{T H D}$ of the no-load current waveform is a suitable performance measure for PWM techniques employed in high power drives [10]. The weighted total harmonic distortion factor $V_{W T H D}$ of the line voltage waveform is an equivalent quantity, independent of motor parameters. The variation in $V_{W T H D}$ with $M$ for different values of $N$ is shown for CSVS, BBCS, BSS and AZCS in Figs. 15a-d, respectively.

A comparison of the $V_{W T H D}$ characteristics of the different strategies is presented in Figs. $16 a$ and $b$. The performances of CSVS, BSS and AZCS, all with a nominal pulse number of 9, are compared in Fig. 16a. Fig. 166 shows a comparison of the performances of CSVS and BBCS, both with $P_{N O M}=15$, and those of BSS and AZCS, both having $P_{N O M}=13$. The bus-clamping PWM strategies perform better than CSVS at higher $\boldsymbol{M}$ including the whole of overmodulation zone. AZCS, despite the lack of QWS in small regions around the zero-crossings of its fundamental voltage, performs best in certain regions at higher $M$. The measured values of $I_{T H D}$ are presented in Figs. $16 c$ and $d$,

which confirm the theoretical predictions in Figs. $16 a$ and $b$, respectively.

\section{Conclusions}

An overmodulation algorithm has been proposed for space vector modulated inverters. Using the proposed algorithm, the extension of operation of four space vector-based synchronised PWM strategies to achieve a higher DC bus utilisation was investigated. Typical pole voltage waveforms, and experimental no-load and full-load current waveforms demonstrated the operation in the overmodulation zone.

It was found that the voltage control characteristics, showing the relationship between the control variable and the fundamental voltage generated, are piecewise linear in overmodulation zone-I, and can be approximated by straight lines in overmodulation zone-11. The voltage control characteristics in the overmodulation zone vary widely with the strategy used and the number of samples per sec- 

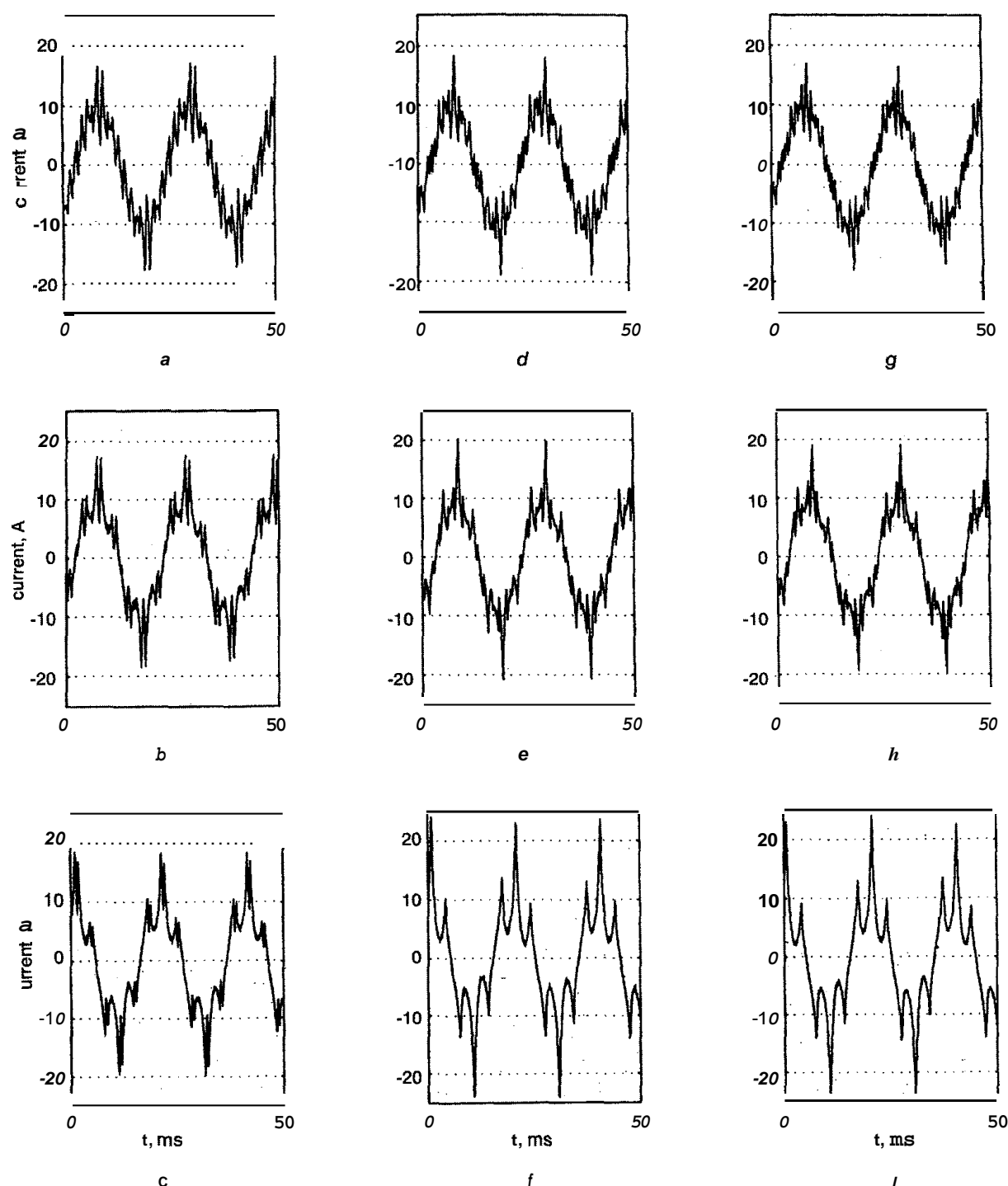

Fig.12 Experimental no-load current waveforms-II $a-C B B C S$ with $N=S$ and $60^{\prime}$ ' clamp, $M=0.92,0.96, M_{M A X}=0.989$ $a-c B B C S$ with $N=5$ and 60" clamp, $M=0.92,0.96, M_{M A X}=0.989$
$d-f$ BBCS with $N=5$ and $30^{\circ}$ clamp, $M=0.92,0.96, M_{M A X}=1.0$ $d-f$ BBCS with $N=5$ and $30 "$ clamp, $M=0.92,0.96$
$g-i$ BSS with $N=6, M=0.92,0.96, M_{M A X}=1.0$

tor $N$ despite the application of the same overmodulation algorithm. While $M=0.907$ is the boundary between circular modulation zone and overmodulation zone-I, and $M=$ 0.952 is the boundary between overmodulation zone-I and overmodulation zone-I1 at high switching frequencies, these boundaries are different for different low switching frequency PWM strategies and also for different values of $\mathrm{N}$. With bus-clamping strategies, operation is possible up to the six-step mode if the strategy has no sample at the centre of the sector and/or the PWM waveform generated is of the 30" clamping type. Operation is not possible up to the six-step mode, whenever the strategy has a sample at the centre and also the PWM waveform generated is of the $60^{\circ}$ clamping type.

The performance of the different strategies has been assessed. The bus-clamping PWM strategies result in lower harmonic distortion than the conventional space vector strategy at higher modulation indices inclusive of the overmodulation zone as shown by theoretical as well as experimental results. These are especially useful in high power drives with short ranges of speed, close to the base speed, as in fan and pump drives.

\section{References}

1 HANDLEY, P.G., and BOYS, J.T.: 'Practical real-time PWM modulators - an assessment',IEE Proc. B, Electr. Power Appl., 1992, 139 (2) pp. 96-102

2 HOLTZ, J.: 'Pulsewidth modulation for electronic power conversion', Proc. IEEE, 1994, 82, (8), pp. 1194-1214

3 HOLMES, D.G.: 'The significance of zero space vector placement for carrier-based PWM schemes', IEEE Trans. Ind. Appl., 1996, 32, (5), pp. $1122-1129$

4 BLASKO, V.: 'Analysis of a hybrid PWM based on modified spacevector and triangle-comparison methods', IEEE Trans. Ind. Appl., 1997,33, (3), pp. 756 764

5 HAVA, A.M KERKMAN, R J and LIPO, T A - 'Simple analytical and graphical methods for carrier-based PWM-VSI drives', IEEE Trans. Power Electron., 1999, 14, (1), pp. 49-61

6 CHUNG, D.-W., KIM, J.-S., and SUL, S.-K.: 'Unified voltage modulation technique for real-time three-phase power conversion', IEEE Trans. Ind. Appl., 1998,34, (2), pp. 374-380

7 YOUM, J.-H., and KWON, B.-H.: 'An effective software implementation of the space-vector modulation', IEEE Trans. Ind Electron., 1999,46, (4), pp. 866-868 

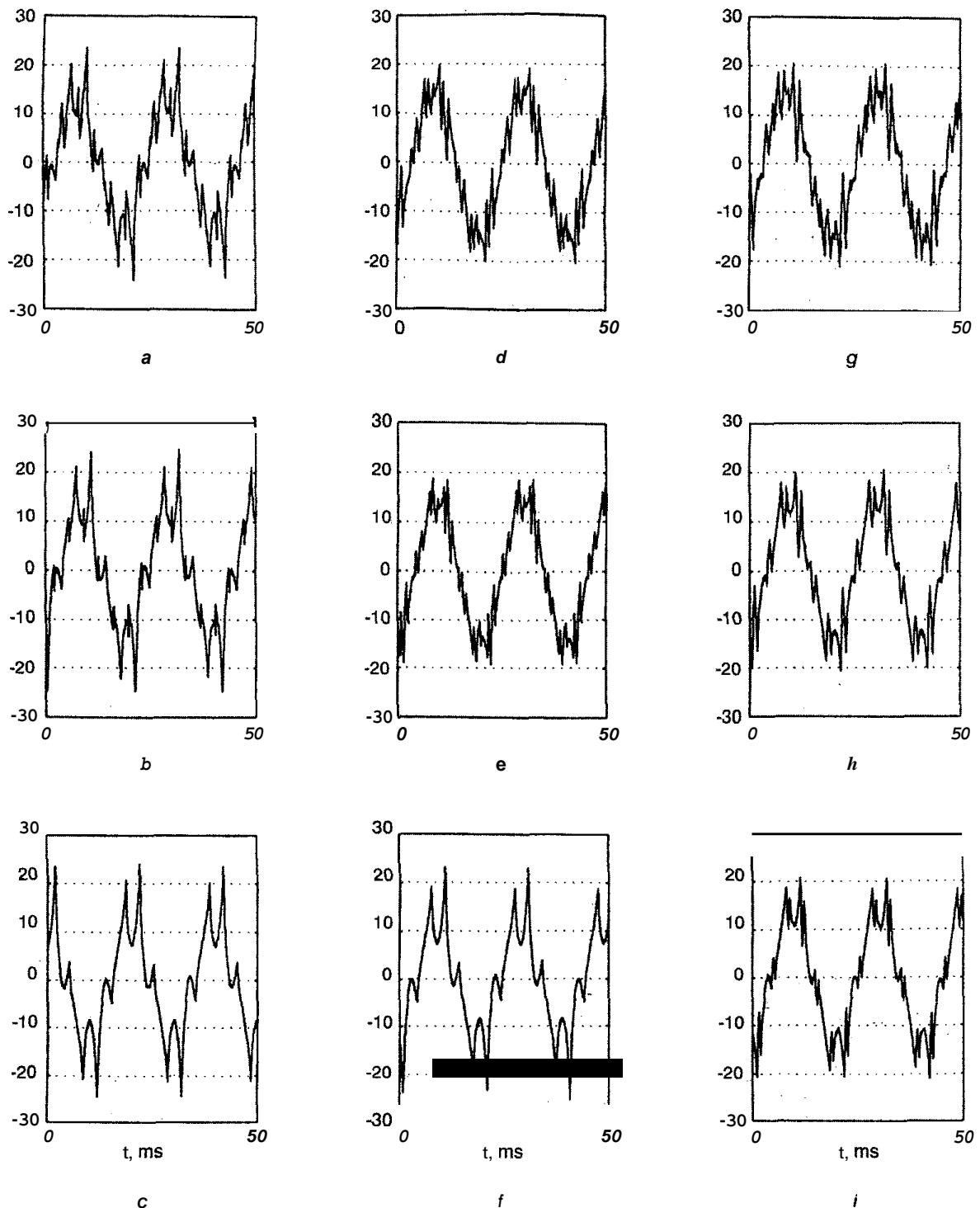

Fig.13 Experimentalfull-loud current wuveform-I $a-C$ CSVS with $N=3, M=0.92,0.96, M_{M A X}=1.0$ df $f$ AZCS with $N=4, M=0.92,0.96, M_{M A X}=1.0$ g-i BSS with $N=4, M=0.92,0.96, M_{M A X}=0.983$

8 NARAYANAN, G., and RANGANATHAN, V.T.: 'Synchronised PWM Strategies based on space vector approach. Part 1: Principles of waveform generation', IEE Proc. B, Electr. Power Appl., 267-275,146, (3)

9 'SynchronisedPWM strategies based on space vector approach. Part 2 : Performance assessment and application to V/f drives', IEE Proc. $B$, Electr. Power Appl.,146, (3), pp. 276281

10 STEMMLER, H.: 'High-power industrial drives', Proc. IEEE, 1994, 82, (8), pp. 12661286

11 HAVA, A.M., LIPO, T.A., and KERKMAN, R.J.: 'Carrier-based PWM-VSI overmodulation strategies: analysis, comparison and design', IEEE Trans. Power Electron., 1998, 13, (4), pp. 674-689

12 KAURA, V.: 'A new method to linearise any triangle-comparisonbased PWM by reshaping the modulation command', IEEE Trans. Ind Appl., 1997, 33, (5), pp. 1254-1259

13 BOLOGNANI, S., and ZIGLIOTTO, M.: 'Novel digital continuous control of SVM inverters in the overmodulation range', IEEE Trans. Ind Appl, 1997,33 (2) pp. 525-530

14 HOLTZ, J., LOTZKAT, W., and KHAMBADKONE, A.: 'On continuous control of PWM inverters in the overmodulationrange including the six-step mode', IEEE Trans. Power Electron., 1993, 8, (4), pp. $546-553$

15 LEE, D.-C., and LEE, G.-M.: 'A novel overmodulationtechnique for space-vector PWM inverters', IEEE Trans. Power Electron., 1998, 13, (6), pp. 1144-1151 

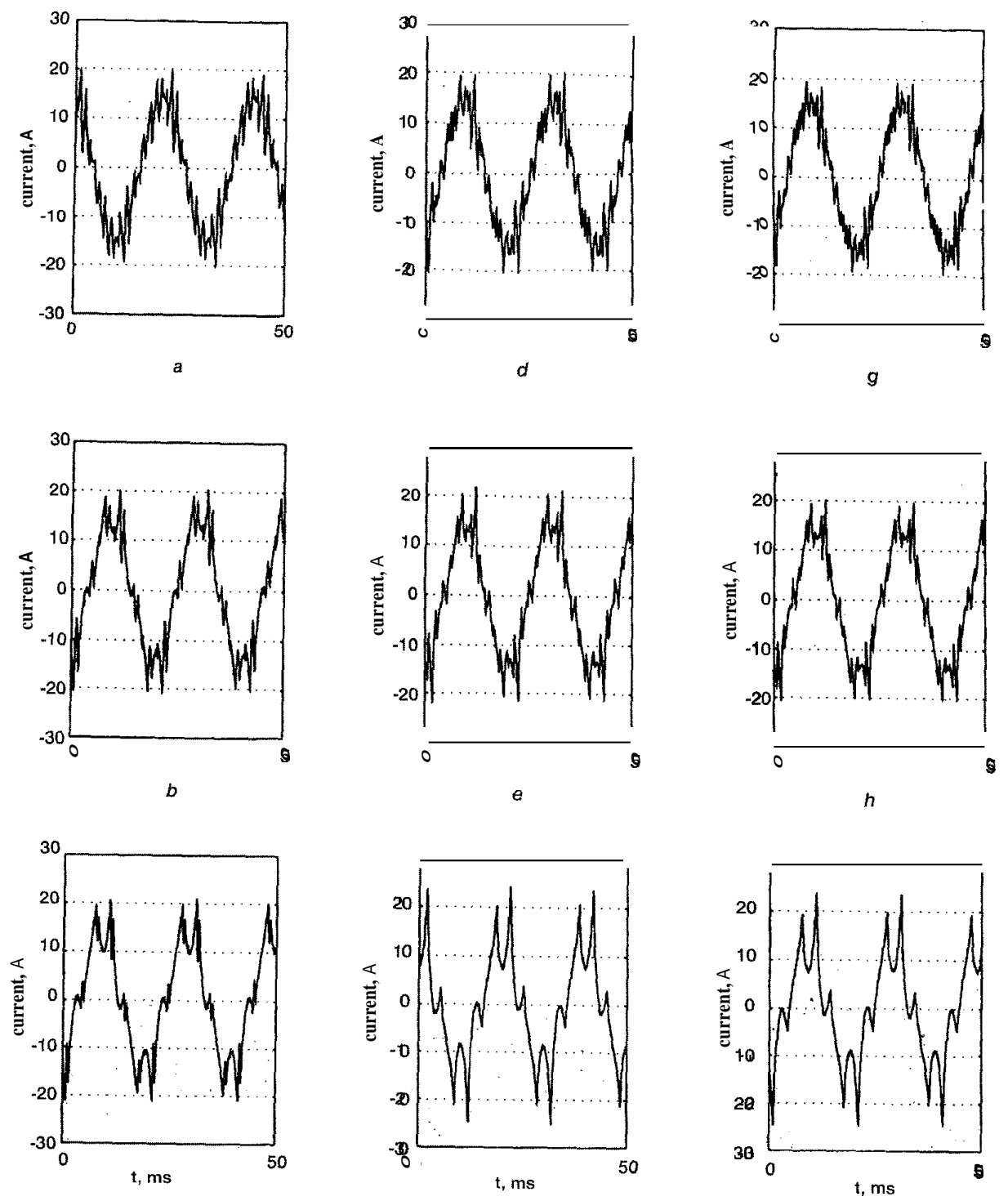

Fig. 14 Experimental full-load current waveforms-II a $c$ BBCS with $N=5$ and $60^{\circ}$ clamp, $M=0.92,0.96, M_{M A X}=0.989$ d $f$ BBCS with $N=5$ and $30^{\circ}$ clamp, $M=0.92,0.96, M_{M A X}=1.0$ $g-i$ BSS with $N=6, M=0.92,0.96, M_{M A X}=1.0$ 

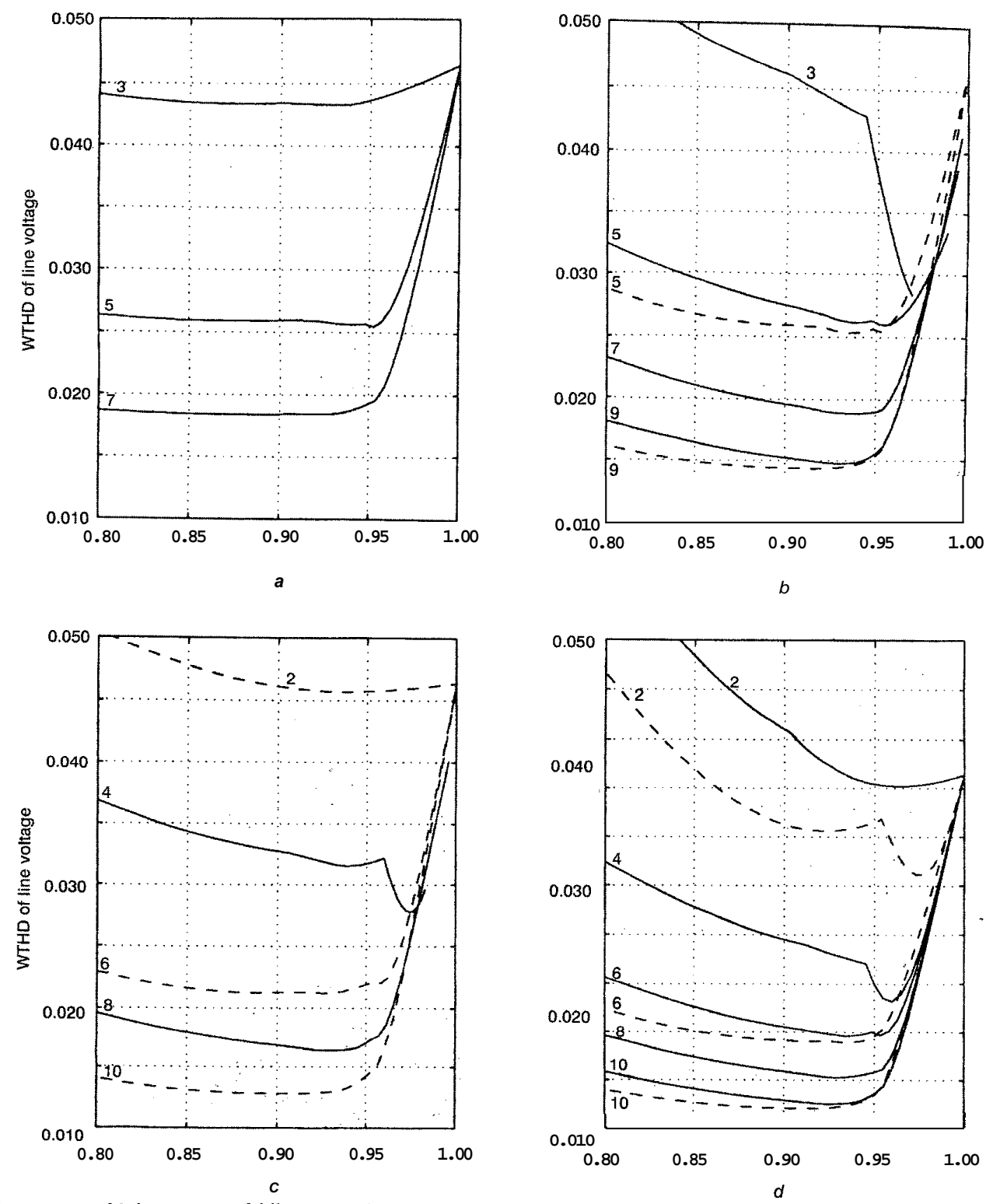

Fig.15 $V_{W T H D}$ against $M$ characteristics of different strategies $a$ CSVS with $N=3,5,7 ; P_{N O M}=3 N$

$h$ BECS with $N=3,5,7,9 ; P_{\text {NOM }}=2 N+1$

$c$ BSS with $N=2,4,6,8,10 ; P_{N O M}=2 N+1$
$d$ AZCS with $N=2,4,6,8,10 ; P_{N O M}=2 N+1$

$-\quad 60 "$ clamp
$---\quad 30^{\prime \prime}$ clamp 

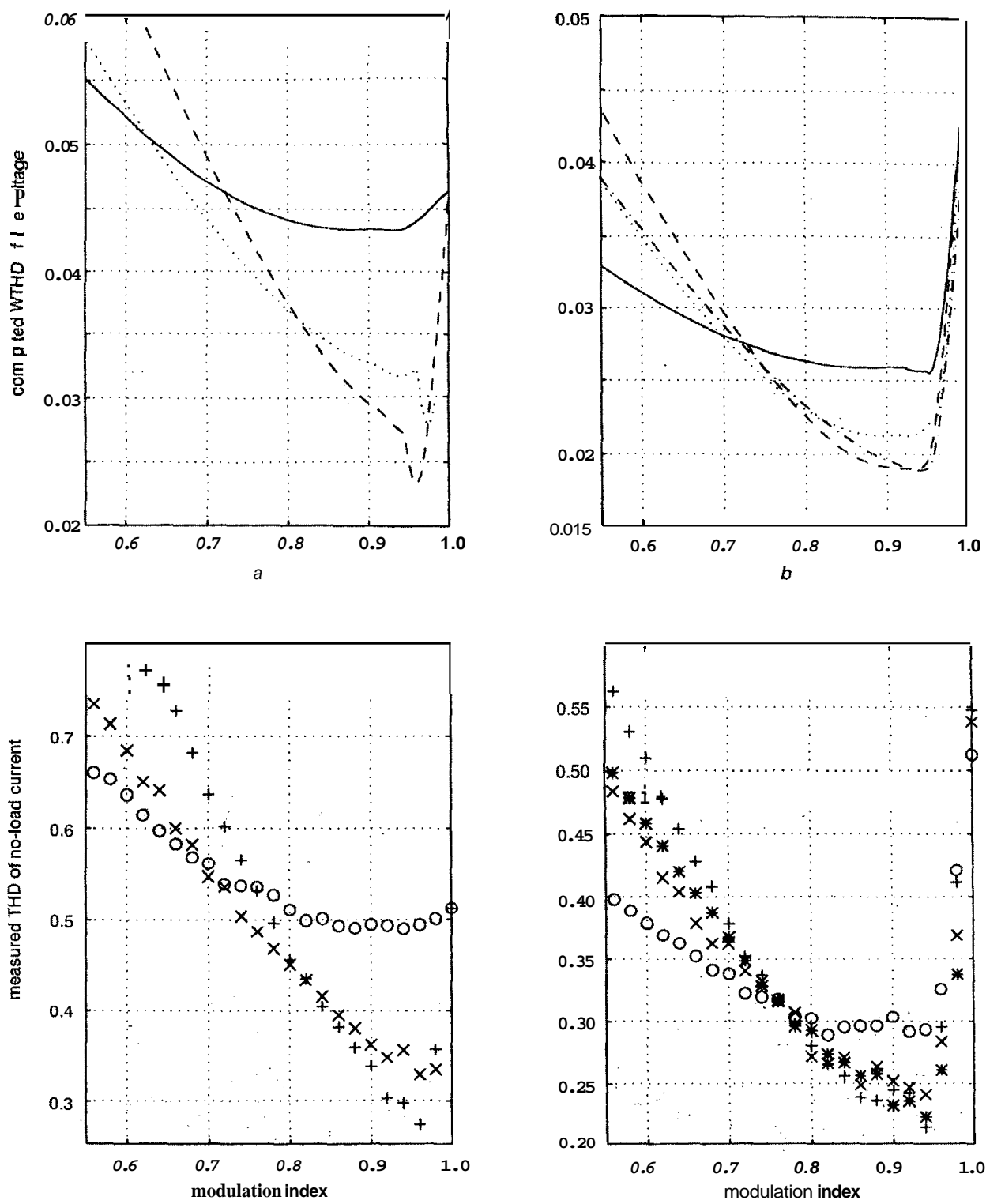

Fig.16 Comparison of performance of different strategies a Computed $V_{\text {WTHD }}$ against $M$
CSV with

- AZCS with $P_{N O M}=9$

.......... BSS with $P_{\text {NOM }}=9$

$b$ Computed $V_{\text {HTHD }}$ against $M$

CSVS with $P_{N O M}=15$

AZCS with $P_{\text {NOM }}=13$

BCS with $P$ M $=13$

$c$ measured $I_{T H P}$ against $M$

O CSVS with $P_{\text {NOM }}=9$

+ AZCS with $P_{N O M}=9$

x BSS with $P_{N O M}=9$

$d$ measured $I N$ against $M$

OCSVS with $P_{N O M}=15$

+ AZCS with $P_{N O M}^{N O M}=13$

$\mathrm{X}$ BSS with $P_{N O M}=13$

$*$ BBCS with $P_{N O M}=15$ 